\title{
A Survey on Snake Species Identification using Image Processing Technique
}

\author{
Pranalini Joshi \\ Department of IT \\ Engineering \\ Zeal College of \\ Engineering \& Research, \\ Pune -411041 \\ Maharashtra, India
}

\author{
Dhanesh Sarpale \\ Department of IT \\ Engineering \\ Zeal College of \\ Engineering \& \\ Research, Pune - \\ 411041 \\ Maharashtra, India
}

\author{
Rohan Sapkal \\ Department of IT \\ Engineering \\ Zeal College of \\ Engineering \& Research, \\ Pune -411041 \\ Maharashtra, India
}

\author{
Apeksha Rajput \\ Department of IT \\ Engineering \\ Zeal College of \\ Engineering \& \\ Research, Pune - \\ 411041 \\ Maharashtra, India
}

\begin{abstract}
Snakes play essential role in the eco-system and are not indispensable. Deforestation are disturbing the habitat of snakes. Identification of the snake is important in planning treatment in certain areas of the world, but is not always possible. Snake bite is more common, local knowledge not may be sufficient to recognize the snake. Attempting to catch or kill the offending snake also puts one at risk, and generally is not recommended. To prevent this, proposed system is introduce to tackle this problem which can identify accurately the species of snake.
\end{abstract}

\section{General Terms}

Snake species identification, Object Detection, Moving Object Detection, Object Classification, YOLO algorithm, Image processing .

\section{Keywords}

Snake species identification, Snake species recognition, Image processing technique, Object detection, Real-time object detection, Object classification, Snake species.

\section{INTRODUCTION}

Snake are the reptiles animals which are useful to keep Ecosystem healthy. When snakes are encounter to anyone usually people try to kill or catch them which are fatal or dangerous. Aim of this project is to prevent snake from getting killed and also to save the human life. As Human being do not have knowledge about snake species, they consider snake as very dangerous reptile animal. As Snake species are identified visually , which may leads to inaccurate result for identification. Incorrect snake identification from the observable visual traits is a major reason of death of people resulting from snake bites. So far no automatic classification method has been proposed to distinguish species of snakes. To avoid it, this project is introduced. This project is an application which will capture image or video and classify snake species using image processing.

Five most common poisonous snakes found in Maharashtra are Indian Cobra, Malabar Pit Viper, Russell's Viper, Saw scaled Viper and Krait. Through this project, we will identify different parameters from snake images for automated snake classification studies. For real-time implementation of the classifier we are using YOLO [YOU ONLY LOOK ONCE] algorithm for image processing. This system finds application in wild life studies, analysis of snake bites and in management of snake population.
The morbidity and death due to snakebite is high in many parts of the Maharashtra even with the availability of anti-venin. It is observed that the patients are taken into account in identifying snake species, however, most of the doctors are not trained to identify the taxonomy of the snake, so accuracy of detection in practice is very low. This system will give the accurate result of snake species within one minute. The result of proposed system will be accurate that it will help doctor and patient to find which species required for preparation of anti-venin. So we can reduce the death caused due to snakebites.

\section{LITERATURE SURVEY}

India has been recognised as having the highest snakebite death in the world. Most of the time death is caused due to unavailability of antivenin \& victims failed to reach the hospital in time [6]. Another major cause is failed to recognized snake species. Snake poison contains hemotoxins which is useful in blood related health issues(Blood cancer ,high blood pressure)

Maharashtra has reported highest incidence as 70 bites per 100000 population and mortality of 2.4 per 100,000 per year. The identification of snake species is crucial for optimal clinical management, as it allows doctors to choose the appropriate treatment, anticipate complications, and thereby reducing death.[4]

Snake bite deaths occurred mostly in rural areas $(97 \%)$, were more common in males $(59 \%)$ than females $(41 \%)$, and peaked at ages $15-29$ years $(25 \%)$ and during the monsoon months of June to September. This proportion represents about 45,900 annual snakebite deaths nationally $(99 \%)$ or an annual age standardized rate of 4.1/100,000 $(99 \%)$, with higher rates in rural areas (99\%)[10].

\section{PROPOSED SYSTEM}

Proposed system uses two modules, one of them is object detection and another one is classification. Object detection module will detect snake as an object from input and classification module will classify object into appropriate species of snake.

Object detection module , uses YOLO (YOU ONLY LOOK ONCE) algorithm[2].YOLO uses deep learning and convolution neural networks ( $\mathrm{CNN})$ for object detection, as the name indicates it only needs to "see" each image once. This allows YOLO to be one of the fastest detection algorithms and can detect objects in real time (up to 30 FPS). 
In recent years, the field of object detection has seen enormous in progress. Object detection is nothing but the task of identifying objects in an image and drawing bounding boxes around them.

Previous approaches of object detections are proposed with pipeline structure that have separate stages in a sequence. This causes the less accuracy and more time delay for result. Rather than passing multiple sub-images of objects, YOLO passes the whole image to the deep learning system once. As a result, YOLO algorithm gives bounding boxes as well as object category classification at a time.

YOLO architecture is same as FCNN (fully convolutional neural network). The working of YOLO is that it subdivides the image into an $\mathrm{NxN}$ grid, or more specifically consider as a $7 \times 7$ grid. Each grid cell, also known as an anchor, represents a classifier which is responsible for generating $\mathrm{K}$ bounding boxes around objects whose ground truth centre falls within that grid cell and classifying it as the correct object.

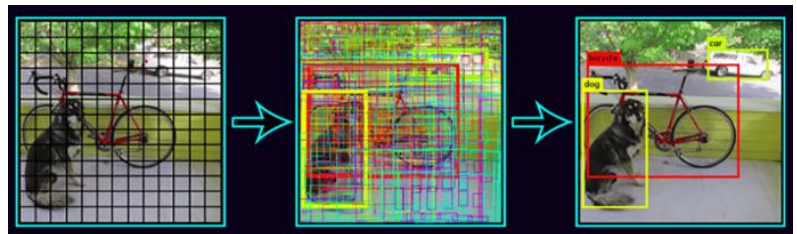

Fig 1 . Working of YOLO[2]

The working of YOLO algorithm can be done via a 2-stage process[2]

The first stage involved creating tens of thousands of proposals. They are nothing but specific rectangular areas on the image also known as bounding boxes, of what the system believed to be object-like things in the image. The bounding box proposal could either be around an actual object in an image or not, and filtering this out was the objective of the second stage.

In the second stage, an image classifier would classify the subimage inside the bounding box, and the classifier would say if it was of a particular object type or simply a non-object or background.

This is done in a single pass through the image at presumption time. Thus, the joint detection and classification leads to better increment the learning objective (the loss function) as well as real-time performance. The result of YOLO algorithm is shown in Fig 2:

\section{Fig 2. Result of YOLO algorithm[2]}

In YOLO each grid cell predicts $\mathrm{B}$ bounding boxes and confidence scores for those boxes. These confidence scores reflect how confident the model is that the box contains an object and also how correct it thinks the box is that it predicts. Formally confidence can be defined as $\operatorname{Pr}($ Object $) *$ IOU(Intersection Over Union). If no object present in that cell, the confidence scores should be zero. Otherwise the confidence score should be equal to the intersection over union (IOU) between the predicted box and the ground truth.

Each bounding box consists of 5 predictions: $\mathrm{x}, \mathrm{y}$, $\mathrm{w}, \mathrm{h}$, and confidence. The $(\mathrm{x}, \mathrm{y})$ coordinates represent the center of the box relative to the bounds of the grid cell. The width and height are predicted relative to the whole image. Finally the confidence prediction represents the IOU between the predicted box and any ground truth box. Each grid cell also predicts $\mathrm{C}$ conditional class probabilities, $\operatorname{Pr}$ (Classi |Object). These probabilities are conditioned on the grid cell containing an object. It only predict one set of class probabilities per grid cell, irrespective of the number of boxes B.

At test time, multiply the conditional class probabilities and the individual box confidence predictions, which gives classspecific confidence scores for each box.

$\operatorname{Pr}($ Classi $\mid$ Object $) * \operatorname{Pr}($ Object $) * \mathrm{IOU}=\operatorname{Pr}($ Classi $) * \mathrm{IOU}$

Then next working is done by $\mathrm{CNN}$ (Convolutional Neural Network) model[11]. The convolutional layer is the core building block of a CNN. In this detection system CNN has 24 convolutional layers which are followed by two fully connected layers.Here input will be an image to the first convolutional layer. The convoluted output is obtained as an activation map. The filters applied in the convolution layer extract significant features from the input image to pass further. Each filter shall give a different component to aid the correct class prediction. To reduce the number of parameter pooling layers are used. It helps in extracting features.

The output layers of $\mathrm{CNN}$ are fully connected layers. Where the input from the other layers is planned and sent so as the transform the output into the number of classes as chosen by the network.

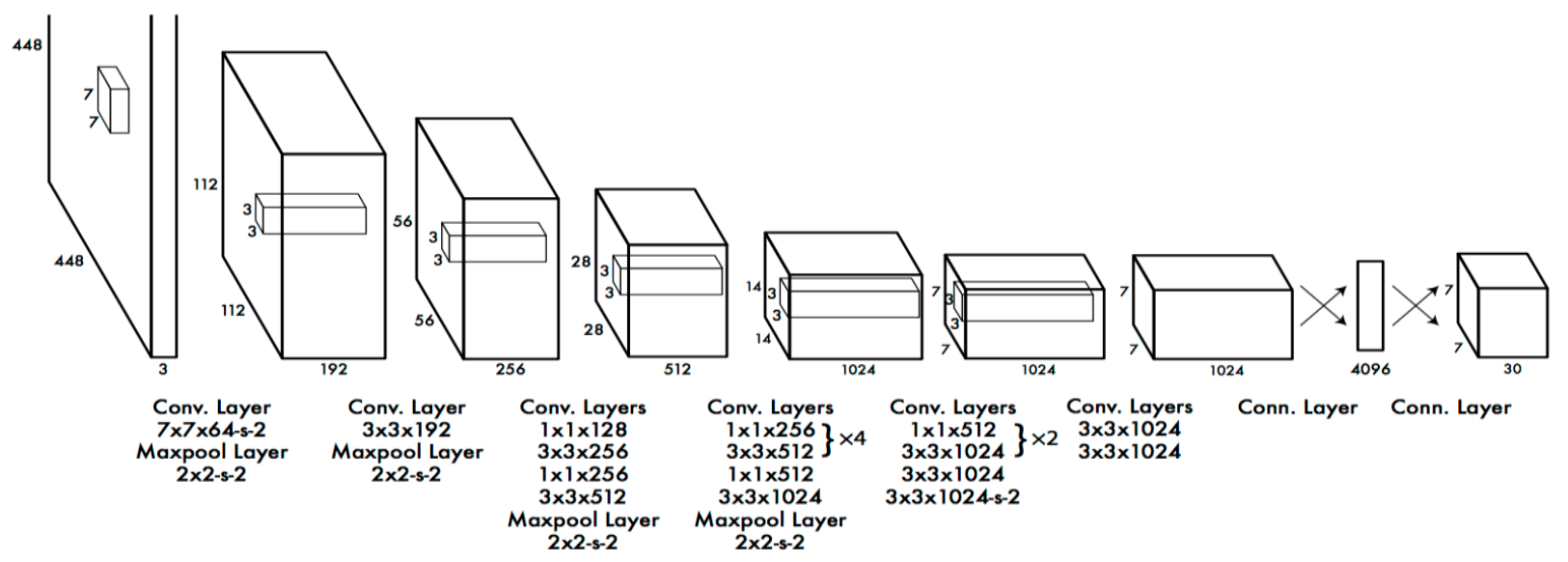

Fig 3. CNN Network Architecture [11] 
One training cycle is completed in a single forward and backward pass. CNN has achieved a large decrease in error rate. CNN is more efficient in terms of memory and complexity.Convolutional Neural Networks take advantage of local spatial coherence in the input (often images), which allow them to have fewer weights as some parameters are shared. By using YOLO algorithm, real time object detection with less time is possible and using CNN classifier snake species classification with more accuracy is possible.

\section{ADVANTAGES OF PROPOSED \\ 4. SYSTEM \\ 1. Time can be reduced as YOLO algorithm is used. \\ 2. Accurate snake species recognition. \\ 3. More accurate due to image processing than visual} identification.

\section{FUTURE SCOPE OF SYSTEM}

For features like image extraction, deep learning, deep neural networks are very effective but these system had time delay for training layers with simple hardware. The usage of CNN's are motivated by the fact that they can capture / are able to learn similar features from an image /video at different levels similar to a human brain.

From this user can get accurate identification of snake species in minimum time. In future scope if snake bite is happened then system will send first aid to user and also inform to nearest emergency health care center. So this system can save snake species which are becoming rare.

\section{CONCLUSION}

The Real time object detection YOLO algorithm is time efficient so it takes less time to detect snake as a object.

Proposed system will identify snake species accurately. Proposed system also help to reduce snake bite causalities. A dataset of snake species is collected, and converted to extract

the taxonomy based feature and image snake dataset.

The taxonomy based snake species dataset can be used as a reference data for classifier to utilize and compare the observations of the snake species.

YOLO algorithm will effectively detect snake as an object and CNN classifier algorithm will distinguish object into appropriate species of snake.

\section{ACKNOWLEDGMENTS}

Greatly acknowledge the Zeal College of Engineering \& Research ,Pune for encouraging for the project on

'Snake species identification using image processing technique'. We express our deep gratitude to Dr. S. A. Ubale(Head of Information Technology department) for her unending support to carry out this work.

\section{REFERENCES}

[1] S.R. Balaji,Dr. S. Karthikeyan, "A Survey On Moving Object Tracking Using Image Processing”, $11^{\text {th }}$ IEEE conference 2017,469-474.

[2] Joseph Redmon, Santosh Divvala,Ross Grishick, Ali Farhadi,"You Only Look Once:Uified, Real-time Object Detection",University of Washington,IEEE conference 2016.

[3] N. Dalal and B. Triggs. "Histograms of oriented gradients for human detectionproblem: Computer vision algorithms for recognising object in artwork and in photographs.", arXiv preprint arXiv:1505.00110,2015.

[4] Hindustan time : "Maharashtra Records more snake bite cases in India 2017",Nov 19,2017.

[5] World Health Organization," snake bites are now global health proprity",Aug 04,2017.

[6] The Hindu :"India tops in death due to snake bites",Aug $04,2017$.

[7] R.B Girshick, Fast R-CNN. CoRR,abs/1504.08083,2015.

[8] S. Gould, T. Gao and D. Koller. "Region-based segmentation and object detection. In advances in Neural Information Processing System, pages 655-663,2009.

[9] K. Lenc and A. Vedaldi "R-CNN minus", arXiv preprint arXiv:1506.06981,2015.

[10]X. Zhou, K. Yu, T. Zhang, T. Huang, "Image classification using super-vector coding of local image descriptors", ECCV, 2010.

[11] Nadia Jmour ; Sehla Zayen ; Afef Abdelkrim "Convolutional neural networks for image classification" IEEE

,2018. 